\title{
Induction of myxospores in Stigmatella aurantiaca (myxobacteria): inducers and inhibitors of myxospore formation, and mutants with a changed sporulation behaviour
}

\author{
Klaus Gerth, ${ }^{*} \dagger$ Rolf Metzger and Hans Reichenbach $\dagger$ \\ Institut für Biologie II der Universität Freiburg, Lehrstuhl für Mikrobiologie, Freiburg, Germany
}

(Received 22 September 1992; revised 9 November 1992; accepted 4 January 1993)

\begin{abstract}
In a screening programme, many new compounds were found that induced myxospore formation in Stigmatella aurantiaca strain Sg a1. The most efficient compounds were indole and indole derivatives, e.g. 3-methylindole. With an inducing concentration of $0.07 \mathrm{mM}$, this compound was about 2000 times more efficient than glycerol. Indole compounds, which have also been found as natural metabolites of myxobacteria, are thus candidates for autoinducers within the fruiting bodies. Mutants were isolated that were resistant to particular inducers. They often showed cross-resistance to many other inducers. By the sporulation response of three different mutants, the inducers could be classified into four groups. Strains resistant to inducers of, e.g. group I were still sensitive to inducers of groups II and III, and vice versa. This classification was supported by newly discovered inhibitors of induced sporulation. These inhibitors, oxindole and pyrrole, were specific for inducers of group $I$. The grouping is further supported by differences in the kinetics of myxospore formation. We postulate that $S$. aurantiaca has three inducer-specific, independent receptors.
\end{abstract}

\section{Introduction}

In the biology of the myxobacteria, two forms of morphogenesis can be distinguished. A cooperative morphogenesis results in the formation of fruiting bodies and a cellular morphogenesis which occurs in nature within these maturing fruiting bodies then converts vegetative cells into myxospores. (Rosenberg, 1984; Shimkets, 1990; Reichenbach \& Dworkin, 1992).

Since the discovery that in Myxococcus xanthus, myxospores could be artificially induced by the addition of certain chemicals to vegetatively growing cells in liquid culture, it has been possible to separate fruiting body formation from myxospore formation, and to study the latter under strictly controlled conditions (Dworkin \& Gibson, 1964).

Stigmatella aurantiaca $\mathrm{Sg}$ al is another well established and optimized system for the study of induced myxospore formation (Gerth \& Reichenbach, 1978), and is even more versatile with respect to inducing compounds

* Author for correspondence.

$\dagger$ Present address: Gesellschaft für Biotechnologische Forschung, GBF, Arbeitsgruppe Mikrobielle Sekundärstoffe, Mascheroder Weg 1, D-3300 Braunschweig, Germany. Tel. 5316181 433; fax 5316181515. than Myxococcus xanthus. Myxospore formation may be induced not only by glycerol, but also by monovalent cations such as sodium or potassium (Reichenbach \& Dworkin, 1970), and even an increase of the incubation temperature to $41^{\circ} \mathrm{C}$ may trigger cellular morphogenesis (Gerth \& Reichenbach, 1978). For the closely related species Stigmatella erecta another inducer, 1,2-propanediol, has also been described (Galvan \& de Castro, 1988).

The objective of this work was to screen for new inducing chemicals, to gain insights into which structural elements are required for inducing compounds, and to learn something about the targets of the inducers and about signal transduction. For this purpose, we isolated mutants of $S$. aurantiaca that displayed altered sporulation behaviour with respect to their response to certain inducers, and which allowed us to study cross-reactions between the various inducers.

\section{Methods}

Experimental organism and culture conditions. Stigmatella aurantiaca strain Sg al has been described previously (Gerth \& Reichenbach, 1978), as have the details of the general conditions for cultivation and myxospore induction. For the isolation of mutants, a medium was developed which allowed high plating efficiency. PT(Sg)-agar consisted 
of $1.5 \%$ Casitone (Difco), $0 \cdot 1 \% \mathrm{MgSO}_{4} .7 \mathrm{H}_{2} \mathrm{O}, 0.2 \% \mathrm{CaCl}_{2} .2 \mathrm{H}_{2} \mathrm{O}$, $1.5 \%$ agar (all w/v), adjusted to $\mathrm{pH} 7.0$ with $\mathrm{KOH}$ and sterilized by autoclaving. Casitone liquid medium (CLM) was used for suspension cultures and consisted of $1 \%(\mathrm{w} / \mathrm{v})$ Casitone and $0.2 \% \mathrm{MgSO}_{4} .7 \mathrm{H}_{2} \mathrm{O}$. The $\mathrm{pH}$ was 6.8 and was not adjusted.

Screening for inducing chemicals. All chemicals used were of analytical grade. Stock solutions ( $300 \mathrm{mM}$ ) were prepared by dissolving the compounds in CLM; the solutions were sterilized by autoclaving. Compounds which were not completely soluble were used as wellmixed suspensions. The compounds were tested in a concentration range of 150 to $0.01 \mathrm{mM}$ by performing a series of $1: 2$ dilution steps. The total volume of each culture was $1 \mathrm{ml}$. The test-tubes with the cultures were incubated for $2 \mathrm{~h}$ on a reciprocal shaking water bath at $32{ }^{\circ} \mathrm{C}$. Sporulation was ascertained by microscopic examination.

Screening for compounds that prevented glycerol-induced myxospore formation. The procedure was as described above for induction, with the modification that the sporulation inducer glycerol $(130 \mathrm{~mm})$ was first added, followed immediately by substances to be tested for inhibition of induction.

Screening for inducing chemicals. If not otherwise stated, the wildtype strain $\mathrm{Sg}$ a I was used for induction experiments. The bacteria were cultivated in $50 \mathrm{ml}$ CLM for $3 \mathrm{~d}$. Then $10 \mathrm{ml}$ portions were pipetted into sterile $100 \mathrm{ml}$ Erlenmeyer flasks, and the supplements were added. The cultures were then incubated for $2 \mathrm{~h}$ at $32^{\circ} \mathrm{C}$ on a rotary shaker.

The following chemicals were tested for myxospore induction or specific inhibition of glycerol-induced myxospore formation and were found negative for both.

Amino acids: cysteine, ethionine, histidine, homoarginine, methionine, norvaline, tryptophan, tyrosine, valine.

Inorganic ions: $\mathrm{BaCl}_{2}, \mathrm{CaCl}_{2}, \mathrm{CsCl}, \mathrm{MgSO}_{4}$.

Organic acids: $m$-aminobenzoic acid, $p$-aminobenzoic acid, ascorbic acid, barbituric acid, 4-chlorobenzoic acid, glutaric acid, glyoxylic acid, $m$-hydroxybenzoic acid, indole-3-acetic acid, indole-2carboxylic acid, iodoacetic acid, maleic acid, malonic acid, $\alpha$ naphthyl acetate, nicotinic acid, 2-oxoglutarate, phthalic acid, pyruvic acid, quinolinic acid, succinic acid, succinic anhydride, thiobarbituric acid, urea, valeric acid.

Organic bases: adenine, adenosine, 5-bromouracil, cAMP, cGMP, cIMP, cUMP, cytosine, diphenylamine, ethanolamine, guanine, hydroxylamine, imidazole, 1-methyladenine, p-phenylenediamine, putrescine, spermidine, thymine, tryptamine, xanthine.

Miscellaneous: 4-amino-2-methylnaphthol, $m$-anisidine, betaine, cetyltrimethylammonium bromide, 1,2-dihydroxybenzene, 1,4-dihydroxybenzene, 2,2'-dipyridyl, $N$-ethylmaleimide, fructose, 8-hydroxyquinoline, inositol, 3,3'-methylene-bis(4-hydroxycoumarin), 2-methyl-1,5-naphthoquinone, ouabain, papaverine, 1,10-phenanthroline, ribose, sucrose, sulphanilamide, tetrachloro-o-benzoquinone, toluene.

Mutagenesis. Bacterial cultures $(50 \mathrm{ml})$ in mid-exponential phase were sedimented by centrifugation at 10000 r.p.m. and $10^{\circ} \mathrm{C}$. The pellets were suspended in $40 \mathrm{ml}$ Tris/ $\mathrm{HCl}$ buffer $(25 \mathrm{mM}$-Tris, $15 \mathrm{~mm}$ $\mathrm{MgSO}_{4}, \mathrm{pH} 7 \cdot 4$ ). After addition of $150 \mu \mathrm{g} N$-methyl- $N$-nitroso- $N^{\prime}-$ nitroguanidine (NTG, Serva) dissolved in $0.1 \mathrm{ml}$ acetone, the cultures were incubated for $1 \mathrm{~h}$ at $30^{\circ} \mathrm{C}$. The cells were then suspended in fresh medium, incubated overnight, and plated on PT agar.

Selection of mutants that were no longer inducible to form myxospores. The tendency of myxospores to clump together and form settling aggregates (Gerth \& Reichenbach, 1978) was used to select for cells that continued to grow vegetatively even in the presence of an inducing compound. Four hours after addition of the inducer, $10 \mathrm{ml}$ of the NTG-treated culture was centrifuged for $2 \mathrm{~min}$ at 5000 r.p.m. and room temperature, to sediment the myxospores, and the supernatant, containing myxospores and vegetative cells, was used to inoculate $50 \mathrm{ml}$ fresh CLM. This procedure was repeated several times and led to an enrichment of non-inducible cells in the supernatant. After plating, mutants could be isolated which were no longer inducible by the compound used during the selection procedure.

Selection of mutants hypersensitive to glycerol induction. Glycerol at a final concentration of $60 \mathrm{~mm}$ (about half the optimum concentration for induction) was added to a culture that had been mutagenized with NTG. After incubation overnight, the vegetative cells were lysed within $4 \mathrm{~h}$ by the addition of $150 \mathrm{~mm}$-glycine. The surviving myxospores were recovered by centrifugation, washed with distilled water and resuspended in fresh growth medium. This selection step was repeated about 10 times before cloning.

Determination of the kinetics of myxospore development. The $\mathrm{OD}_{623}$ of induced cultures was determined every $10 \mathrm{~min}$ in an Eppendorf spectrophotometer, using a $1 \mathrm{~cm}$ cuvette. Because of changes at the cell surface, the differentiating cells began to form aggregates of increasing size which caused a decrease in the $\mathrm{OD}_{623}$ of the sporulating culture. Myxospore formation was always checked by microscopic observation.

\section{Results}

\section{Inducers of myxospore formation}

About 150 different chemicals were tested for their potential to induce myxospores or to inhibit glycerolinduced myxospore formation in $S$. aurantiaca.

Induction of myxospore formation could be demonstrated for 39 compounds (Table 1). Among these where numerous amino acids, alcohols, aromatic and cationic compounds. While many of them are natural intermediates of bacterial metabolism, the optimum concentrations needed for induction were usually between 50 and $200 \mathrm{~mm}$ and thus beyond physiological levels. Some of the compounds have been reported as inducers for Myxococcus xanthus as well, e.g. ethylene glycol, glycerol, 2-phenylethanol, 2-propanol and n-butanol (Sadler \& Dworkin, 1966).

One compound of particular interest was 3-methylindole, which was about 2000 times more active than glycerol and acted at a concentration of $10 \mu \mathrm{g} \mathrm{ml}^{-1}$. Concerning the kinetics of myxospore formation, we could distinguish between a large group of inducers effective within $60 \mathrm{~min}$, and a smaller group of compounds that did not produce typical myxospores within $2 \mathrm{~h}$ of their addition (Table 1).

Glucose showed a completely irregular inducing behaviour. The bacteria continued to grow vegetatively for hours, and myxospores were discovered only 18-24 h after addition of the sugar (Table 1).

\section{Inhibitors of induced myxospore formation}

Inhibitors that prevented glycerol-induced myxospore formation in $\mathrm{Sg}$ al were also found during the screening (Table 2). For example, oxindole at a final concentration 
Table 1. Compounds which induce myxospore formation in S. aurantiaca

\begin{tabular}{|c|c|c|c|c|c|c|c|c|}
\hline \multirow[b]{2}{*}{ Group } & \multirow[b]{2}{*}{ No. } & \multirow[b]{2}{*}{ Compound } & \multirow{2}{*}{$\begin{array}{l}\text { Optimum } \\
\text { concentration } \\
\text { (mM) }\end{array}$} & \multicolumn{3}{|c|}{$\begin{array}{l}\text { Myxospores produced } \\
\text { with mutant: }\end{array}$} & \multicolumn{2}{|c|}{ Shape change after: } \\
\hline & & & & M 13 & M 50 & M 16 & $60 \mathrm{~min}$ & $120 \mathrm{~min}$ \\
\hline \multirow[t]{27}{*}{ I } & 1 & Glucose & 350 & - & + & & & $24 \mathrm{~h}$ \\
\hline & 2 & Ethylene glycol & 190 & - & + & + & + & \\
\hline & 3 & Methionine & 150 & - & + & + & + & \\
\hline & 4 & DMSO & 135 & - & + & + & + & \\
\hline & 5 & Glycerol & 130 & - & + & + & + & \\
\hline & 6 & Asparagine & 120 & - & + & + & + & \\
\hline & 7 & Glutamine & 120 & - & + & + & + & \\
\hline & 8 & Hydroxyproline & 100 & - & + & + & + & \\
\hline & 9 & Isoleucine & 100 & - & + & + & + & \\
\hline & 10 & Leucine & 100 & - & + & + & + & \\
\hline & 11 & Proline & 100 & - & + & + & + & \\
\hline & 12 & Threonine & 90 & - & + & + & + & \\
\hline & 13 & Alanine & 80 & - & + & + & + & \\
\hline & 14 & 2,2-Dimethylbutane & 80 & - & + & + & + & \\
\hline & 15 & $\mathrm{KCl}$ & 80 & - & + & + & + & \\
\hline & 16 & Lysine & 75 & - & & & + & \\
\hline & 17 & Tris & 60 & - & + & + & + & \\
\hline & 18 & $\mathrm{NaCl}$ & 55 & - & + & + & + & \\
\hline & 19 & Arginine & 50 & - & + & + & + & \\
\hline & 20 & Phenylalanine & 50 & - & + & + & + & \\
\hline & 21 & Choline & 32 & - & + & + & + & \\
\hline & 22 & 2-Dimethylaminoethanol & 30 & - & + & + & + & \\
\hline & 23 & Triethanolamine & 30 & - & + & + & + & \\
\hline & 24 & tert-Butylamine & 20 & - & + & + & + & \\
\hline & 25 & Uracil & 20 & - & + & + & + & \\
\hline & 26 & Theophylline & 12 & - & + & + & + & \\
\hline & 27 & Nalidixic acid & 4 & - & + & + & + & \\
\hline \multirow[t]{5}{*}{ II } & 28 & 2-Phenylethanol & $6 \cdot 5$ & + & - & - & & + \\
\hline & 29 & 2-Phenylpropanol & $4 \cdot 0$ & + & - & - & & + \\
\hline & 30 & Indoline & $2 \cdot 4$ & + & - & - & & + \\
\hline & 31 & Benzene & 1.8 & + & - & - & & + \\
\hline & 32 & 2-Methylindole & $0 \cdot 4$ & + & - & - & & + \\
\hline \multirow[t]{2}{*}{ III } & 33 & tert-Butanol & 90 & + & + & - & & + \\
\hline & 34 & 2-Propanol & $12 \cdot 5$ & + & + & - & & + \\
\hline \multirow[t]{5}{*}{ IV } & 35 & n-Butanol & 4 & + & + & + & + & \\
\hline & 36 & Indole & $0 \cdot 1$ & + & + & + & + & \\
\hline & 37 & 3-Methylindole & 0.07 & + & + & + & + & \\
\hline & 38 & Benzildioxime & $0 \cdot 3$ & - & - & - & & \\
\hline & 39 & 2,3-Benzofuran & $0 \cdot 12$ & - & - & - & & \\
\hline
\end{tabular}

Table 2. Inhibitors of chemically induced myxospore formation

\begin{tabular}{lccccc}
\hline \hline & & \multicolumn{4}{c}{ Inducer } \\
\cline { 2 - 6 } & $\begin{array}{c}\text { Concn } \\
(\mathrm{mM})\end{array}$ & $\begin{array}{c}\text { Glycerol } \\
(130 \mathrm{mM})\end{array}$ & $\begin{array}{c}\text { 2-Phenylethanol } \\
(6.5 \mathrm{mM})\end{array}$ & $\begin{array}{c}\text { tert-Butanol } \\
(90 \mathrm{mM})\end{array}$ & $\begin{array}{c}\text { Indole } \\
(0 \cdot 1 \mathrm{mM})\end{array}$ \\
\hline Inhibitor & $0 \cdot 3$ & - & + & + & - \\
Oxindole & $3 \cdot 6$ & - & + & + & - \\
Pyrrole & 5 & - & + & + & \\
2-Propanol & 20 & - & + & + \\
tert-Butanol & & & & + & \\
\hline \hline
\end{tabular}

of only $0.3 \mathrm{~mm}$ prevented induction with $130 \mathrm{mM}$ glycerol. 2-Propanol and tert-butanol, which are themselves inducers (Table 1), inhibited glycerol-induced sporulation if added at 5 and $20 \mathrm{mM}$, respectively, below the concentrations at which they were themselves inducing. 
Table 3. Test of cross-resistance of sporulation-defective mutants to alternative inducers

\begin{tabular}{llllllll}
\hline \hline & & \multicolumn{5}{c}{ Response to alternative inducers of myxospore formation } \\
\cline { 3 - 7 } Mutant & $\begin{array}{l}\text { Selected for } \\
\text { resistance to: }\end{array}$ & Glycerol & $\mathrm{NaCl}$ & DMSO & Proline & 2-Phenylethanol & tert-Butanol \\
\hline M 13 & Glycerol & - & - & - & - & + & + \\
M 46 & NaCl & - & - & - & - & + & + \\
M 43 & Proline & - & - & - & - & + & + \\
M 35 & DMSO & - & - & - & - & + & + \\
M 50 & 2-Phenylethanol & + & + & + & + & - & + \\
M 16* & tert-Butanol & + & + & + & + & - & + \\
\hline \hline
\end{tabular}

* M 16 was derived from mutant M 50.

None of these inhibitors could, however, prevent myxospore induction with the full range of inducers (Table 2). Thus, induction by 2-phenylethanol or tertbutanol was exceptional in that it could not be blocked by the glycerol-induction-specific inhibitors; even the concentrations needed for induction remained unchanged in the presence of the various inhibitors.

\section{Mutants resistant against a specific inducing compound}

Starting with the wild-type strain Sg a1, mutants were enriched and isolated which could no longer be induced to form myxospores by the following inducers at their optimum concentrations: glycerol, $\mathrm{NaCl}$, proline, DMSO, 2-phenylethanol and tert-butanol. Mutants which could not be induced to form myxospores are called 'resistant' in this paper.

Mutants resistant to glycerol, $\mathrm{NaCl}$, proline and DMSO could easily be isolated (Table 3 ). The selection of mutants resistant to the inducer 2-phenylethanol caused some problems. A characteristic feature of this compound, as well as of some other inducers, was that myxospores showed a much reduced tendency to form sedimentable aggregates, which is a prerequisite for enrichment. Nevertheless, the method described was eventually successful.

In contrast, myxospores induced with tert-butanol clumped very well, but we were still not able to find resistant mutants. However, further mutagenization of mutant M 50, which is resistant to 2-phenylethanol, yielded tert-butanol-resistant strains. The characteristics of some of the isolated mutants are summarized in Table 3.

Mutants resistant to glycerol, $\mathrm{NaCl}$, proline and DMSO were cross-resistant among themselves. For example, mutant M 46, which was resistant to $55 \mathrm{mM}$ $\mathrm{NaCl}$, could no longer be induced with $130 \mathrm{~mm}$-glycerol (for the optimum inducing concentrations, see Table 1).

Surprisingly, all these mutants could still be induced by 2-phenylethanol and tert-butanol. On the other hand, mutants M 50 and M 16, which were selected for their resistance to 2-phenylethanol and tert-butanol, respectively, still produced myxospores in the presence of, for example, glycerol (Table 3). While mutant M 50, resistant to 2-phenylethanol, could be induced by tertbutanol, M 16 was also resistant to tert-butanol.

The concentration optima of the alternative inducers needed for the induction of resistant mutants were determined (Table 4). Glycerol-resistant M 13 behaved like the wild-type strain, with the exception of indole, for which the required concentration was increased by $525 \%$. Phenylethanol-resistant mutant $M 50$ was as sensitive as the wild-type strain to glycerol. The required concentrations of tert-butanol, 2-propanol and indole were, however, increased by between 20 and $60 \%$.

\section{Isolation and characterization of mutants that could be induced with suboptimal concentrations of glycerol}

Mutant M 20 was isolated as a strain with increased sensitivity to glycerol compared to the wild strain. Optimum sporulation was obtained at $40 \mathrm{~mm}$-glycerol, compared with $130 \mathrm{~mm}$ with the original strain. Simultaneously, the mutant was more sensitive to many other inducers (Table 5). Exceptions were 2-phenylethanol and tert-butanol to which the strain was only slightly more sensitive (by $20-30 \%$ ). Also, the concentration of tert-butanol necessary to inhibit glycerol induction was reduced in the same manner: $6 \mathrm{mM}$ tertbutanol was sufficient to inhibit M 20 compared with $20 \mathrm{~mm}$ with the original strain.

\section{Kinetics of indole-induced myxospore formation in mutants $M 50$ and $M 13$}

Morphological changes of phenylethanol-resistant M 50 and glycerol-resistant M 13 cultures were followed by measuring changes in the optical density of the cultures after the addition of $0.2 \mathrm{~mm}-$ and $0.6 \mathrm{~mm}$-indole, respectively (Fig. 1). While mutant M 50 showed kinetics typical for glycerol-induced myxospore formation, with a drastic decrease in $\mathrm{OD}_{623}$ after $30 \mathrm{~min}, \mathrm{M} 13$ behaved 
Table 4. Effect of resistance to a particular inducer on the optimal concentration with alternative inducers

\begin{tabular}{lccccc}
\hline \hline & \multicolumn{4}{c}{ Myxospore induction with the alternative inducers } \\
\cline { 2 - 6 } Strain & Glycerol & 2-Phenylethanol & tert-Butanol & 2-Propanol & Indole \\
\hline Wild-type & $100 \%$ & $100 \%$ & $100 \%$ & $100 \%$ & $100 \%$ \\
M 13 & - & $100 \%$ & $100 \%$ & $100 \%$ & $625 \%$ \\
M 50 & $100 \%$ & - & $122 \%$ & $158 \%$ & $137 \%$ \\
\hline \hline
\end{tabular}

Table 5. Comparison of optimal inducer concentrations for induction in the wild-type and in mutant $M 20$ (selected for higher sensitivity to glycerol)

\begin{tabular}{lccc}
\hline & \multicolumn{2}{c}{ Optimal concentration (mM) } & Sensitivity increase \\
\cline { 2 - 4 } Inducer & Wild strain & M 20 & \begin{tabular}{c} 
(\%) \\
\hline Glycerol
\end{tabular} \\
\cline { 2 - 4 } Ethylene glycol & 130 & 40 & 69 \\
DMSO & 190 & 65 & 66 \\
NaCl & 135 & 45 & 67 \\
Indole & 55 & 27 & 51 \\
2-Phenylethanol & $0 \cdot 1$ & $0 \cdot 035$ & 65 \\
tert-Butanol & $6 \cdot 5$ & $4 \cdot 6$ & 29 \\
& 90 & 70 & 22 \\
\hline \hline
\end{tabular}

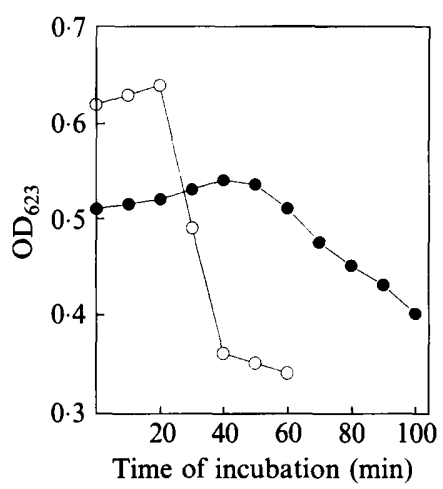

Fig. 1. Kinetics of indole-induced myxospore formation. Mutants M 50 (O) and M 13 (O) were induced by the addition of $0.2 \mathrm{mM}$ and 0.6 mM-indole, respectively. The $\mathrm{OD}_{623}$ of the cultures was measured every $10 \mathrm{~min}$.

like the wild strain induced with 2-phenylethanol with the typical time delay and reduced aggregation.

\section{Discussion}

S. aurantiaca strain $\mathrm{Sg}$ al can be induced to produce myxospores by a wide variety of different chemicals (Table 1), and mutants can be isolated that are resistant to certain inducers. Resistance of a mutant to a certain inducer does not necessarily mean that the mutant has lost its potential for cellular morphogenesis altogether, for the mutations do not always interfere with the sequence of metabolic events that follow the inducing stimulus. Rather, the cells may have lost their sensitivity towards certain inducers.

We have shown that there are several classes of inducer-resistant mutants. With the help of the mutants M 13, M 50 and M 16, most of the inducers of myxospore formation in $\mathrm{Sg}$ a 1 can be classified into four groups (Table 1). A mutant isolated as resistant against any one inducer of one group is cross-resistant to all other inducers of this group, without ever having had contact with these compounds (Table 3). All mutants of this class are, however, inducible by the other groups of inducers, and vice versa. An exception is M 16, which cannot be induced by compounds of group II because M 16 derives from (phenylethanol-resistant) M 50 and thus is a double mutant. In no case was the resistance due to a reduced sensitivity to the respective compound, as drastically increased concentrations still could not induce the developmental process.

An explanation for our experimental results could be that the cells possess three different types of inducerspecific receptors. Inducers of group I (Table 1), which we shall call the glycerol group, act by interference with receptor I. Inducers of group II, the 2-phenylethanol group, induce through receptor II. We believe that these two receptors are independent from each other, because the inducing concentrations needed for compounds of the reciprocal group were not changed by the mutations (Table 4). This interpretation is also supported by the behaviour of glycerol-hypersensitive mutant M 20, which shows a uniformly increased sensitivity of about $65 \%$ only to inducers of group I (Table 5). The situation is somewhat more complicated for the inducers of the tertbutanol group III (Table 1).

We suggest that inducers of group III interact with the phenylethanol receptor II and, at the same time, with a tert-butanol-specific receptor III. This hypothesis would explain why mutants resistant against tert-butanol could only be isolated from the mutant M 50, which was already defective in receptor II, as starting from the wildtype strain or from $M 13$, a double mutation in receptors II and III would be necessary in order to obtain tertbutanol resistance. Also, higher concentrations of tert- 
butanol and 2-propanol were required for the induction of mutant M 50, because this mutant is defective in receptor II, so that the inducers could act only via the remaining receptor III.

We believe that inducers of group IV are also acting on two different receptors. Thus, higher indole concentrations were required to induce (glycerol-resistant) mutant M $13 \quad(525 \%)$ and (phenylethanol-resistant) mutant M $50(37 \%)$ compared to the wild-type strain (Table 4). We postulate a strong affinity of group IV inducers to receptor I, and a lower one to receptor II.

All inhibitors of myxospore induction found by us (Table 2) were specific for receptor I. Induction with optimum concentrations of compounds of this group, e.g. glycerol, was prevented by oxindole or pyrrole in extremely low concentrations (in relation to the inducer concentrations). Those compounds thus appear to have a high affinity to receptor I. Induction by $6.5 \mathrm{~mm}-2$ phenylethanol (receptor II) or $90 \mathrm{mM}$-tert-butanol (receptors II and III) was not influenced. This result also demonstrates that binding alone is not sufficient to activate the cells to produce myxospores.

2-Propanol and tert-butanol were also inhibitors of receptor I when applied in sub-inducing concentrations. So these compounds seem to interact with receptor I although they are not able to induce through this receptor. The sensitivity of (glycerol-hypersensitive) mutant $\mathbf{M} 20$ to this inhibition of glycerol induction is also increased by $70 \%$. The fact that induction by $0.1 \mathrm{~mm}$-indole is also inhibited by 2-propanol and tertbutanol (Table 2) supports the suggestion that group IV compounds act preferentially by interference with the glycerol receptor. At higher indole concentrations $(0.7 \mathrm{mM})$ the glycerol-resistant mutant M 13 could successfully be induced via receptor II (Table 4).

Our hypothesis of receptor-specific induction is supported by a completely different set of data. When we compared the kinetics of myxospore formation using different inducers, we observed that with inducers of group I and group IV morphological changes began after $30 \mathrm{~min}$ and were complete after $60 \mathrm{~min}$, while with inducers of groups II and III they started only after $60 \mathrm{~min}$ and were complete after $2 \mathrm{~h}$ (Table 1). Not only was induction by type I inducers faster than by type II inducers, but the cell aggregates formed during cell conversion were also much bigger. The kinetics of myxospore induction with indole (Fig. 1) were investigated with the phenylethanol-resistant mutant $M 50$ and the glycerol-resistant mutant M 13. With M 50, which is supposedly defective in receptor II, the kinetics were identical with those of the wild type. Here induction occurs by interaction with receptor I. With $\mathbf{M} 13$, which is probably defective in receptor $I$, induction can only occur via receptor II, and in fact the time needed for myxospore formation was longer, as is typical for inducers of group II.

In addition to the inducers already known, many new inducing compounds were discovered in the course of our screening. Most of them (about $69 \%$ ) belong to group I. The inducing concentrations of these compounds vary in wide limits. While with the best known inducer, glycerol, concentrations as high as $130 \mathrm{~mm}$ were required, nalidixic acid, an inhibitor of DNA synthesis, induced myxospore formation in $S$. aurantiaca at $4 \mathrm{mM}$.

At the moment it is not possible to define a common functional group for the inducers acting on receptor I. It appears, however, that hydrogen bonding can be excluded, because $150 \mathrm{~mm}$-urea or $75 \mathrm{~mm}$-dimethyl formamide, both well known for their hydrogen bonding, neither stimulate nor inhibit induction. Ionic and dipole interactions between inducer and receptor are more likely to be important. Inducers of group II are much more efficient with respect to concentration than group I inducers. They are more lipophilic, and hydrophobic interactions could be essential for induction. Inducers of group III and IV are interacting with two different receptors at the same time.

Compounds 38 and 39, benzildioxime and 2,3benzofuran, respectively, could not be classified in any of the established groups, because only the wild-type strain could be induced by them. Also, glucose is not a typical inducer, as growth continued for a long time after its addition. We feel that the high concentrations required for the inducing effect can best be explained by the production of acids as a consequence of sugar degradation. The accumulating protons may be the actual inducers and may act like other cationic compounds via receptor I.

Indole and its derivatives are the most interesting effectors (inhibitor and inducers) we could detect so far. In contrast to most other compounds, they work at very low concentrations and are at the same time also intermediates of myxobacterial metabolism. Several indole derivatives have meanwhile been isolated from various species of myxobacteria in our studies of the secondary metabolites from myxobacteria (unpublished results). Perhaps the natural inducer of cellular morphogenesis within the maturing fruiting body is a compound of the indole family.

We want to thank the Deutsche Forschungsgemeinschaft and the Sonderforschungsbereich 46 'Molekular Grundlagen der Entwicklung' for their financial support.

\section{References}

Dworkin, M. \& Gibson, S. M. (1964). A system for studying microbial morphogenesis: rapid formation of microcysts in Myxococcus xanthus. Science 146, 243-244.

Galvan, A. \& De Castro, F. (1988). Myxospore induction by several 
agents, myxospore resistance against several environmental factors. Boletin de la Real Sociedad Espanola de Historia Natural, Seccion Biologica 84, 95-104.

GeRTH, K. \& REICHENBaCh, H. (1978). Induction of myxospore formation in Stigmatella aurantiaca (Myxobacterales). Archives of Microbiology 117, 173-182.

REICHENBACH, H. \& DWORKIN, M. (1970). Induction of myxospore formation in Stigmatella aurantiaca (Myxobacterales) by monovalent cations. Journal of Bacteriology 101, 325-326.

ReICHENBaCH, H. \& Dworkin, M. (1992). The myxobacteria. In The
Prokaryotes, 2nd edn, chapter 188, pp. 3416-3487. Edited by A. Balows, H. G. Trüper, M. Dworkin, W. Harder \& K. H. Schleifer. New York: Springer Verlag.

ROSENBERG, E. (1984). Myxobacteria development and cell interactions. New York, Berlin, Heidelberg \& Tokyo: Springer Verlag.

SADLER, W. \& DWORKIN, M. (1966). Induction of cellular morphogenesis in Myxococcus xanthus. Journal of Bacteriology 91, $1520-1525$.

ShimkeTs, L. J. (1990). Social and developmental biology of the myxobacteria. Microbiological Reviews 54, 473-501. 